\title{
About uncertainties in practical salinity calculations
}

\author{
M. Le Menn \\ French Hydrographic and Oceanographic Service (SHOM), SHOM, CS 92803, 29228 Brest Cedex 2, France
}

Received: 22 September 2009 - Published in Ocean Sci. Discuss.: 28 October 2009

Revised: 21 September 2011 - Accepted: 29 September 2011 - Published: 17 October 2011

\begin{abstract}
In the current state of the art, salinity is a quantity computed from conductivity ratio measurements, with temperature and pressure known at the time of the measurement, and using the Practical Salinity Scale algorithm of 1978 (PSS-78). This calculation gives practical salinity values $S$. The uncertainty expected in PSS-78 values is \pm 0.002 , but no details have ever been given on the method used to work out this uncertainty, and the error sources to include in this calculation. Following a guide published by the Bureau International des Poids et Mesures (BIPM), using two independent methods, this paper assesses the uncertainties of salinity values obtained from a laboratory salinometer and Conductivity-Temperature-Depth (CTD) measurements after laboratory calibration of a conductivity cell. The results show that the part due to the PSS-78 relations fits is sometimes as significant as the instrument's. This is particularly the case with CTD measurements where correlations between variables contribute mainly to decreasing the uncertainty of $S$, even when expanded uncertainties of conductivity cell calibrations are for the most part in the order of $0.002 \mathrm{mS} \mathrm{cm}^{-1}$. The relations given here, and obtained with the normalized GUM method, allow a real analysis of the uncertainties' sources and they can be used in a more general way, with instruments having different specifications.
\end{abstract}

\section{Introduction}

Salinity is one of the fundamental quantities for which measurement or computation is essential to determine the fundamental properties of seawater. In the current state of the art, salinity is computed from conductivity ratio measurements, with temperature and pressure known at the time of measurement, and using the Practical Salinity Scale algorithm

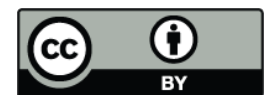

Correspondence to: M. Le Menn (marc.lemenn@shom.fr) defined by Perkin and Lewis (1980). This algorithm gives practical salinities from the ratio of the electrical conductivity of seawater at $15^{\circ} \mathrm{C}$ related to that of a standard potassium chloride solution $(\mathrm{KCl})$.

In practice, in laboratories, salinity is computed from a conductivity ratio measured with salinometers calibrated with IAPSO standard seawater bottles whose salinity and conductivity ratio at $15^{\circ} \mathrm{C}$ or $K_{15}$ are known. At sea, instruments are equipped with conductivity cells calibrated and linearized in seawater baths whose temperature is controlled and measured with great accuracy and whose salinity is determined by salinometers.

The World Ocean Circulation Experiments (WOCE) programme suggested that temperature and conductivities could be measured respectively to $0.002^{\circ} \mathrm{C}$ and $0.002 \mathrm{mS} \mathrm{cm}^{-1}$, resulting in a salinity measurement accuracy of \pm 0.002 (Saunders et al., 1991), but no details were given on the method used to work out the uncertainty measurements and which error sources should be included in this calculation. Several Conductivity-Temperature-Depth (CTD) instruments manufacturers propose equipment whose specifications are supposed to fill these criteria. However, which are really the uncertainties obtained on the measurements needed to establish or to check these criteria? A simple addition of uncertainties, as often seen in manuscripts, (cf. Uschida et al., 2008, for example) is incorrect because sensitivities and input quantity correlations must also be taken into account in the calculations.

Since 1995, the Bureau International des Poids et Mesures (BIPM) has published a guide for the evaluation of measurement data and the expression of uncertainty in measurement (GUM) (BIPM, 2008) based on combined variance of input quantities. In 2008, it offered a supplement to the GUM based on the propagation of distributions using a Monte Carlo method (BIPM, 2006). A probability density function is attributed to each input variable of a mathematical relation, and a piece of software works out the output variable of the relation by generating random numbers for each

Published by Copernicus Publications on behalf of the European Geosciences Union. 
input variable. From the results, the software can produce a histogram of the output variable distribution and its variation statistics (mean, standard deviation, etc.). The GUM and Monte Carlo methods are two independent ways to calculate a measurement uncertainty.

The goal of this paper is to assess the uncertainties of practical salinity calculations using these two methods, when salinity is obtained from laboratory salinometer measurements or from CTD measurements after laboratory calibration of conductivity cells.

\section{Uncertainties on salinities calculated from salinome- ter measurements}

Laboratory salinometers are calibrated with IAPSO standard seawater (SSW) bottles distributed by OSIL (www.OSIL.co. uk), which has the international exclusive rights to do so. The ratio $K_{15}$ of the seawater bottle is determined by OSIL and written on each bottle. Then the conductivity cell of a salinometer at the temperature $t$ measures the conductivity $G_{\mathrm{st}}(t)$, so that:

$G_{\text {st }}(t)=K_{15} C(35,15,0) k_{\text {cellst }}$

$C(35,15,0)$ is the conductivity of standard seawater with a salinity of 35 at the temperature of $15^{\circ} \mathrm{C}$ and the pressure of $0 \mathrm{dbar}$. $k_{\text {cellst }}$ is a value adjusted by the salinometer and which is inversely proportional to the cell constant at the time and the temperature of the measurement. For a seawater sample, the cell will measure $G(t)$, so that:

$G(t)=R_{t} r_{t} C(35,15,0) k_{\text {cell }}$

$k_{\text {cell }}$ being the value inversely proportional to the cell constant, at the time and the temperature of the measurement. $R_{t}$ is the ratio displayed by the salinometer. $R_{t}$ is equivalent to $K_{15}$ for the seawater sample. $r_{t}$ is the temperature correction polynomial of the PSS-78, used to compensate for the temperature effect of the sample:

$r_{t}=c_{0}+c_{1} t+c_{2} t^{2}+c_{3} t^{3}+c_{4} t^{4}$

where $c_{0}, c_{1}, c_{2}, c_{3}$ and $c_{4}$ are constants given for the calculation of salinity (Perkin et al., 1980). See Appendix A to obtain the numerical values of the PSS-78 constants. If we call $\delta k_{\text {cell }}$ the ratio $k_{\text {cellst }} / k_{\text {cell }}, R_{t}$ is given by:

$R_{t}=\frac{G(t)}{G_{\mathrm{st}}(t)} \frac{K_{15}}{r_{t}} \delta k_{\mathrm{cell}}$

The relation (4) describes the conductivity ratio displayed by the salinometer and used to calculate the salinity. $G(t)$ and $G_{\text {st }}(t)$ are two quantities correlated by the temperature. The electrical conductivity signal is a function of salinity, temperature and pressure. However, under typical conditions it is admitted (cf. Lueck, 1990) that the variations of this signal are dominated by temperature to about or at least $80 \%$.
So, $G(t)$ and $G_{\mathrm{st}}(t)$ are strongly dependent on the stability of the cell temperature. The other quantities can be considered as being independent. Then, the GUM method applied to this relation gives the combined standard uncertainty $u_{\mathrm{c}}\left(R_{t}\right)$ of the $R_{t}$ measurement:

$$
\begin{aligned}
u_{\mathrm{c}}\left(R_{t}\right)^{2}= & \left(\frac{\partial R_{t}}{\partial G}\right)^{2} u_{G}^{2}+\left(\frac{\partial R_{t}}{\partial G_{\mathrm{st}}}\right)^{2} u_{G \mathrm{st}}^{2}+\left(\frac{\partial R_{t}}{\partial K_{15}}\right)^{2} u_{K_{15}}^{2} \\
& +\left(\frac{\partial R_{t}}{\partial r_{t}}\right)^{2} u_{r_{t}}^{2}+\left(\frac{\partial R_{t}}{\partial \delta k_{\mathrm{cell}}}\right)^{2} u_{\delta k_{\mathrm{cell}}}^{2} \\
& +2 \frac{\partial R_{t}}{\partial G} \frac{\partial R_{t}}{\partial G_{\mathrm{st}}} u_{G} u_{G \mathrm{st}} r_{G, G_{\mathrm{st}}}
\end{aligned}
$$

$u_{G}$ and $u_{G \text { st }}$ are the standard measurement uncertainties of $G$ and $G_{\text {st }}$, and $r_{G, G_{\text {st }}}$ is their estimated correlation coefficient. We can suppose that $u_{G}=u_{G \text { st }}$ because measurements are made with the same instrument. In the case of a cell temperature drift between the moment of calibration with the SSW and the moment the sample is measured (this is often the case), the $G$ and $G_{\mathrm{st}}$ values depend on this drift. They are, then, strongly correlated. $R_{G, G \text { st }}$ can be inferior to 1 , but let us deliberately take the extreme case where $r_{G, G_{\mathrm{st}}}=1 . u_{K_{15}}, u_{r_{t}}$, and $u_{\delta k_{\text {cell }}}$ are respectively the standard measurement uncertainties of $K_{15}, r_{t}$ and $\delta k_{\text {cell }}$. With these elements, the calculation of $u_{\mathrm{c}}\left(R_{t}\right)$ gives:

$$
\begin{aligned}
u_{\mathrm{c}}\left(R_{t}\right)^{2}= & R_{t}^{2}\left[\left(1-\frac{G}{G_{\mathrm{st}}}\right)^{2}\left(\frac{u_{G}}{G}\right)^{2}+\left(\frac{u_{K_{15}}}{K_{15}}\right)^{2}+\left(\frac{u_{r_{t}}}{r_{t}}\right)^{2}\right. \\
& \left.+\left(\frac{u_{\delta k_{\text {cell }}}}{\delta k_{\text {cell }}}\right)^{2}\right]
\end{aligned}
$$

The advantage of measuring a conductivity ratio appears clearly with the minus sign in the first member of this relation, in the same way as measurements are more precise when $G \approx G_{\text {st }}$, i.e. when the salinity of the sample is near the salinity of the seawater standard used to calibrate the salinometer.

The numerical estimation of $u_{\mathrm{c}}\left(R_{t}\right)$ has been made with the specification values of a Guildline Instruments Limited (Ontario, Canada) Portasal salinometer. Portasal is one of the best known salinometers because, according to Guildline, it can "deliver salinity calculations on-board ships with laboratory level accuracy". So, it is interesting to calculate its measurement uncertainties.

$u_{G}$ can be assessed by the specified conductivity resolution of this instrument which is of $3 \times 10^{-4} \mathrm{mS} \mathrm{cm}^{-1}$ at $S=35$ and $t=15^{\circ} \mathrm{C}$ or $G=42.9175 \mathrm{mS} \mathrm{cm}^{-1}$. The measurement range of the Portasal extends from 0.004 to $76 \mathrm{mS} \mathrm{cm}^{-1}$, and its resolution can vary from $r_{\min }=2 \times$ $10^{-4} \mathrm{mS} \mathrm{cm}^{-1}$ to $r_{\max }=5.3 \times 10^{-4} \mathrm{mS} \mathrm{cm}^{-1}$. According to the GUM supplement 1 (BIPM, 2006), with this available information, a right-angled triangular probability density function (pdf) can be assigned to $u_{G}$, with a maximum at the 
value $5.3 \times 10^{-4} \mathrm{mS} \mathrm{cm}^{-1}$. The standard deviation of such a function leads to express $u_{G}$ as:

$u_{G}=\frac{\left(r_{\max }-r_{\min }\right)}{\sqrt{18}}$

This relation gives: $u_{G}=0.75 \times 10^{-4} \mathrm{mS} \mathrm{cm}^{-1}$.

$u_{K_{15}}$ has been estimated by Bacon et al. (2007). According to this paper, the expanded uncertainty of the standard seawater conductivity ratio has been found to be $1 \times 10^{-5}$ with a coverage factor of 2 at the time of manufacture. This value includes the uncertainty due to the $\mathrm{KCl}$ quality used to prepare the reference conductivity according to Bacon et al. (2007). Kawano et al. (2005) demonstrated that a default of quality could include an uncertainty of 0.001 in the value of the standard salinity $S$. As Bacon et al. (2007) is more recent and since this publication has not yet been refuted, we will retain this value to estimate $u_{K_{15}}$. The way in which this uncertainty has been calculated, leads us to choose a Normal pdf to assess $u_{K_{15}}$ and then: $u_{K_{15}}=5 \times 10^{-6}$. It should be noted that the value $1 \times 10^{-5}$ has been recently analysed by members of the Euromet Project 918 (Seitz et al., 2008). According to Seitz et al., this uncertainty value quantifies the current capability of the standard seawater manufacturer to replicate the conductivity of the $\mathrm{KCl}$ solutions in the short term. This work does not quantify the effects of "aging" and the lifetime of the standard seawater bottles and no value is given to quantify long term variations (over several years or decades) in the production of $\mathrm{KCl}$ solutions. Above all, it fixes the limits of metrological standards in terms of long term salinity traceability, which is not taken into account in the usual use of salinometers. In this assessment, we will consider only the results of Bacon et al. (2007).

$u_{r_{t}}$ can be estimated easily by applying the GUM method to the relation (3) which depends only on $t$. This gives:

$u_{r_{t}}=\left(c_{1}+2 c_{2} t+3 c_{3} t^{2}+4 c_{4} t^{3}\right) u_{t}$

$t$ is the temperature of the bath chosen for making the measurements and $u_{t}$ is provided by the stability of this temperature. $t$ is often chosen to be above the ambient temperature or $24^{\circ} \mathrm{C}$. The stability of this temperature is given to be $\pm 0.001{ }^{\circ} \mathrm{C}$. This value is difficult to hold during long periods of time and it has been checked by measurements on the three Portasals of the SHOM laboratory. The standard deviation of these measurements was never less than $0.001{ }^{\circ} \mathrm{C}$ during periods of 1 to $24 \mathrm{~h}$. So: $u_{t}=0.001{ }^{\circ} \mathrm{C}$.

$u_{\delta k_{\text {cell }}}$ represents the variability of the cell constant which is a function of time and temperature. This variability depends a lot on the stability of the temperature and on the humidity of the laboratory. It can be estimated only by recording fluctuations in the value of $R_{t}$ displayed by the salinometer. These fluctuations are random and $\delta k_{\text {cell }}$ follows a Normal pdf with a standard deviation $u_{\delta k_{\text {cell }}}=2 \times 10^{-5}$.

With these elements, $u_{\mathrm{c}}\left(R_{t}\right)$ was computed for different salinities with the GUM and Monte Carlo methods. In order to make estimates according to the Monte Carlo method, the Oracle Crystal Ball software version 11.1.1.1.000, was used in Microsoft Excel 2002. Table 1 summarizes the parameters of the input quantities and the results. It appears that the biggest contribution to the uncertainty in $R_{t}$ comes from the temperature stability via $r_{t}$ variations. The second contribution comes from the uncertainty of the $K_{15}$ ratio.

Finally, the uncertainty on $S$ was calculated using the PSS78 relation:

$S=\sum_{j=0}^{5} a_{j} R_{t}^{j / 2}+\frac{(t-15)}{1+k(t-15)} \sum_{j=0}^{5} b_{j} R_{t}^{j / 2}$

where $k, a_{j}$ and $b_{j}$ are constants given for the calculation of salinity (Perkin et al., 1980). This relation has two input variables: $R_{t}$ and $t . R_{t}$ depends on $t$ throughout the ratio $r_{t}$. The correlation coefficient $r_{R t, t}$ can be calculated. At atmospheric pressure, for $S=35, r_{R t, t}=0.55$, for $S=40$, $r_{R t, t}=0.54$, and for $S=10, r_{R t, t}=0.97$. The combined standard uncertainty $u_{\mathrm{c}}(S)$ of $S$ is then given by the relation:

$$
\begin{aligned}
u_{\mathrm{c}}^{2}(S)= & \left(\frac{\partial S}{\partial R_{t}}\right)^{2} u_{\mathrm{c}}^{2}\left(R_{t}\right)+\left(\frac{\partial S}{\partial t}\right)^{2} u_{t}^{2} \\
& +2 r_{R_{t}, t} \frac{\partial S}{\partial R_{t}} \frac{\partial S}{\partial t} u_{\mathrm{c}}\left(R_{t}\right) u_{t}
\end{aligned}
$$

where:

$$
\begin{aligned}
\frac{\partial S}{\partial R_{t}}= & \left(\frac{a_{1}}{2} R_{t}^{-1 / 2}+a_{2}+\frac{3 a_{3}}{2} R_{t}^{1 / 2}+2 a_{4} R_{t}+\frac{5 a_{5}}{2} R_{t}^{3 / 2}\right) \\
& +\frac{(t-15)}{1+k(t-15)}\left(\frac{b_{1}}{2} R_{t}^{-1 / 2}+b_{2}+\frac{3 b_{3}}{2} R_{t}^{1 / 2}\right. \\
& \left.+2 b_{4} R_{t}+\frac{5 b_{5}}{2} R_{t}^{3 / 2}\right)
\end{aligned}
$$

and,

$$
\frac{\partial S}{\partial t}=\frac{1}{[1+k(t-15)]^{2}}\left(\sum_{j=0}^{5} b_{j} R_{t}^{j / 2}\right)
$$

Table 1 gives the values of $u_{\mathrm{c}}(S)$ obtained with the GUM computation (0.00081) and with a Monte Carlo simulation (0.00085) for the salinity $S=35$. For $S=10$, the same computations give 0.00023 and 0.00025 and for $S=40: 0.00099$ and 0.0011 . These calculations show that relation (10) can be simplified because the contribution of the first term is largely superior to the contribution of the others, and it can be written as:

$u_{\mathrm{c}}(S) \approx\left(\frac{\partial S}{\partial R_{t}}\right)_{\mathrm{c}}^{u}\left(R_{t}\right)$

However, other uncertainties must be taken into account in the calculation of the uncertainty on salinity. Firstly, salinometers must be controlled at standard salinities other than 35 , in order to correct their linearity errors. These errors can be of 0.003 or more at $S=2$, as seen in calibration reports 
Table 1. Parameters of the input quantities used to compute the expanded uncertainty of Guildline Portasal salinometer for $S=35$, by the GUM and the Monte Carlo (M.C.) methods.

\begin{tabular}{|c|c|c|c|c|c|c|c|}
\hline Input quantities & Unit & Pdf & $\begin{array}{l}\text { Value for } \\
S=35\end{array}$ & $\begin{array}{l}\text { GUM standard measurement } \\
\text { uncertainty }\end{array}$ & $\begin{array}{l}\text { Contribution } \\
\quad \text { to } R_{t}\end{array}$ & $\begin{array}{l}\text { Mean value } \\
\text { by M.C. }\end{array}$ & $\begin{array}{c}\text { Standard deviation } \\
\text { by M.C. }\end{array}$ \\
\hline$t$ & ${ }^{\circ} \mathrm{C}$ & Normal & 24 & 0.0010 & & & \\
\hline$r_{t}$ & None & Normal & 1.212266 & 0.000024 & $3.987 E-10$ & & \\
\hline$K_{15}$ & None & Normal & 0.99984 & $5.00 E-06$ & $2.500 E-11$ & & \\
\hline$G_{\mathrm{st}}$ & $\mathrm{mS} \mathrm{cm}^{-1}$ & Right-angled triangle & 52.0153 & $0.75 E-04$ & $0.000 E+00$ & & \\
\hline$G$ & $\mathrm{mS} \mathrm{cm}^{-1}$ & Right-angled triangle & 52.0153 & $0.75 E-04$ & $0.000 E+00$ & & \\
\hline$\delta_{\text {kcell }}$ & None & Normal & 1.21229 & $-8.51 E-07$ & $\sim 4.931 E-13$ & & \\
\hline$R_{t}$ & None & Normal & 0.999862 & 0.000021 & & 0.999848 & 0.000022 \\
\hline$S$ & None & Normal & $\mathbf{3 4 . 9 9 5 0}$ & 0.00081 & & 34.9940 & 0.00085 \\
\hline Linearity correction & None & Normal & 0.0000 & 0.0001 & & & \\
\hline Salinity value of the bottles & None & Right-angled triangle & 0.0000 & 0.00024 & & & \\
\hline \multirow[t]{3}{*}{$\begin{array}{l}\text { PSS-78 fits } \\
\text { PS }\end{array}$} & None & Normal & 0.0000 & 0.0007 & & & \\
\hline & & & & \multirow{2}{*}{\multicolumn{2}{|c|}{ GUM expanded uncertainty: }} & 0.0022 & \\
\hline & & & & & & & 0.0022 \\
\hline
\end{tabular}

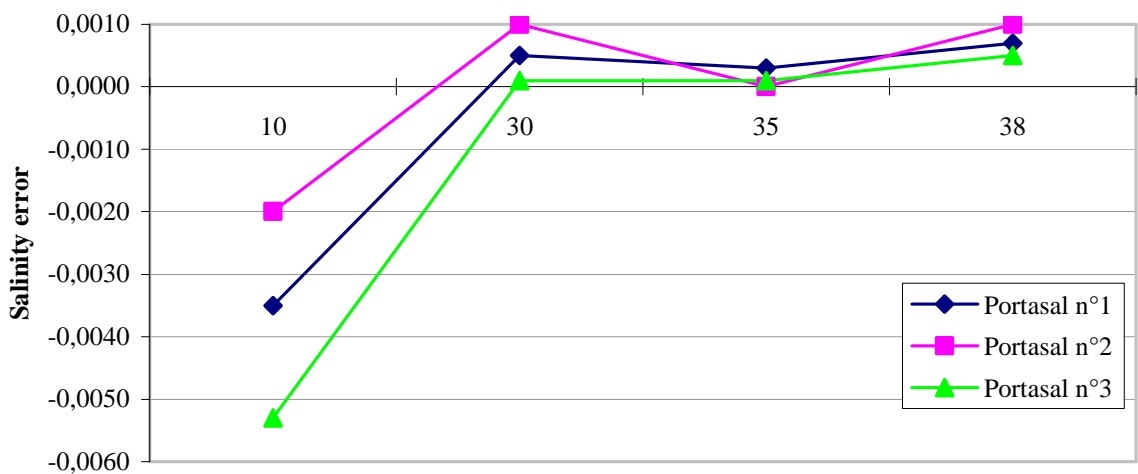

Reference salinity

Fig. 1. Examples of linearity errors measured on three Portasal salinometers after calibration with standard seawater bottles.

made by OSIL on Portasal salinometers (see Fig. 1). Hardware corrections are difficult to make because linearity can be non-uniform in the range of measurements. So, salinity values must be corrected by linear relations on different sub-ranges. These corrections have a standard uncertainty $u_{l}$ which is, at least, equal to the linear regression remainder or 0.0001

Secondly, standard salinity bottles used for the calibration and linearization can show maximum salinity variations of 0.001, for 96 weeks of storage, according to Culkin and Ridout (1998). Resulting from this available information we can assign a rectangular pdf to this uncertainty $\left(u_{\mathrm{sb}}\right)$ according to the GUM. The standard deviation of such a function leads us to express $u_{\mathrm{sb}}$ as: $u_{\mathrm{sb}}=0.001 / \sqrt{3}$.

Thirdly, PSS-78 relations fits have a standard deviation which is of 0.0007 at atmospheric pressure, according to Perkin and Lewis (1980), and of 0.0015 if the pressure term $R_{p}$ is different from 1 . The pdf of this uncertainty ( $\left.u_{\mathrm{PSS}}\right)$ can be considered as being Normal. In the case of salinometers, $u_{\mathrm{PSS}}=0.0007$. $u_{l}, u_{\mathrm{sb}}, u_{\mathrm{PSS}}$ and $u_{\mathrm{c}}(S)$ being independent variables, the expanded uncertainty $U_{S}$ on the salinity, expressed with a coverage factor of 2 to obtain a level of confidence close to $95 \%$, can be written as:

$U_{S}=2 \sqrt{u_{\mathrm{c}}(S)^{2}+u_{l}^{2}+u_{\mathrm{sb}}^{2}+u_{\mathrm{PSS}}^{2}}$

Table 1 gives the values of $U_{S}$ computed with the GUM and Monte Carlo methods and it is the same whatever the method: $U_{S}=0.0022$ for $S=35$ for a Portasal salinometer. Table 2 gives the values obtained for $S=10$ and 40. It appears that the two methods give close results and that for 35 and 40 the expected uncertainty of 0.002 cannot be maintained. The main error sources are the stability of the bath temperature, the linearity of the salinometer, the salinity of the bottles of standard seawater and the PSS-78 itself.

\section{Uncertainties on reference conductivity calculations}

Calibration of conductivity sensors needs the calculation of reference conductivities $C_{\text {ref. }}$. When calibrations are made at atmospheric pressure, $C_{\text {ref }}$ is calculated with the relation: 
Table 2. Standard uncertainties of $R_{t}$ and $S$, and expanded uncertainty on the corrected value of $S$, calculated using the two methods for three different salinities. These values do not take into account possible long term variations in $\mathrm{KCl}$ standard solutions used to adjust standard seawater bottles, or the limits of metrological standards in terms of long term traceability of the salinity.

\begin{tabular}{|c|c|c|c|c|c|c|}
\hline \multirow{2}{*}{$\begin{array}{l}\text { Method } \\
\text { Output quantity }\end{array}$} & \multicolumn{3}{|c|}{ G.U.M. } & \multicolumn{3}{|c|}{ Monte Carlo } \\
\hline & $u_{\mathrm{c}}\left(R_{t}\right)$ & $u_{\mathrm{c}}(S)$ & $U_{S}$ & $u_{\mathrm{c}}\left(R_{t}\right)$ & $u_{\mathrm{c}}(S)$ & $U_{S}$ \\
\hline$S=10$ & $7.0 E-6$ & 0.00023 & 0.0016 & $8.0 E-6$ & 0.00024 & 0.0016 \\
\hline$S=35$ & 0.000021 & 0.00081 & 0.0022 & 0.000022 & 0.00085 & 0.0022 \\
\hline$S=40$ & 0.000024 & 0.00099 & 0.0025 & 0.000027 & 0.0011 & 0.0024 \\
\hline
\end{tabular}

$C_{\text {ref }}=R_{t} r_{t} C(35,15,0)$

where $r_{t}$ is given by the relation (3) and $R_{t}$ is obtained according to Fofonoff and Millard (1983), with a NewtonRaphson iteration and the formula:

$R_{t n+1}=R_{t n}+\left(S-S_{n}\right)\left(\frac{\partial S}{\partial R_{t}}\right)^{-1}$

on condition that we calculate $\left(\partial S / \partial R_{t}\right)$ with the first part of the relation (11).

$C(35,15,0)$ is a constant to which several values have been attributed. According to Culkin and Smith (1980), $C(35,15,0)=42.914 \mathrm{mS} \mathrm{cm}^{-1}$ and according to Poisson (1980), $C(35,15,0)=42.933 \mathrm{mS} \mathrm{cm}^{-1}$. A recent study published by a BIPM working group (CCQM pilot study P111) has attributed the value $42.9104 \mathrm{mS} \mathrm{cm}^{-1}$ to $C(35,15,0)$, after inter-comparisons made by different metrology laboratories (Seitz et al., 2010). In fact, in the case of CTD conductivity sensor calibrations, it does not matter which value is used, provided that the same value is used during data reduction and reference conductivity computations. Most recent instruments are referenced to 42.914 , so, let us take this value in uncertainty calculations, $C(35,15,0)$ being considered as a constant.

The value of $R_{t}$ obtained with the relation (16), depends essentially on $S$ which is measured by a laboratory salinometer and $r_{t}$ depends on $t$. So, we can write that $u_{R t}=$ $\left(\partial R_{t} / \partial S\right) u(S)$ and $u_{r t}=\left(\partial r_{t} / \partial t\right) u_{t}$. However, the computation of the numerical values of $R_{t}$ and $r_{t}$ for different temperatures between 0 and $40^{\circ} \mathrm{C}$, shows that the correlation coefficient $r_{R t, r t}$ is not equal to zero. For $p=0$ and $S=35, r_{R t, r t}=0.53$, for $S=40, r_{R t, r t}=0.56$ and for $S=10, r_{R t, r t}=0.964$. So, $R_{t}$ and $r_{t}$ cannot be considered as two independent variables, and the combined uncertainty of $C_{\text {ref }}$ is given by:

$$
\begin{aligned}
u_{\mathrm{Cref}}= & C(35,15,0)\left[r_{t}^{2}\left(\frac{\partial R_{t}}{\partial S}\right)^{2} u^{2}(S)+R_{t}^{2}\left(\frac{\partial r_{t}}{\partial t}\right)^{2} u_{t}^{2}\right. \\
& \left.+R\left(\frac{\partial R_{t}}{\partial S}\right)\left(\frac{\partial r_{t}}{\partial t}\right) u(S) u_{t} r_{R_{t}, r_{t}}\right]^{1 / 2}
\end{aligned}
$$

$\left(\partial S / \partial R_{t}\right)$ can be calculated with the main part of the relation (11) and $u(S)=U_{S} / 2 ; U_{S}$ being calculated with the relation (14).

$\left(\partial r_{t} / \partial t\right)$ is the polynomial of the relation (8), but in relation (17) $u_{t}$ is the uncertainty of the reference temperatures measured during the calibration of the conductivity sensor. $u_{t}$ depends on reference thermometer calibration uncertainties at fixed ITS-90 points, but also, on the drift of this thermometer between two calibrations, as well as on its selfheating during the measurements and on the stability and uniformity of the calibration bath temperature.

In 2002, the BIPM published a guide (Fellmuth et al., 2002) about uncertainty budgets for the calibration of standard platinum reference thermometers (SPRT) at the fixed ITS-90 points. It takes into account the calibration uncertainties of the fixed points cells themselves, the making of the points, the self-heating errors and the repeatability of the sensors but also, the non-uniqueness of the scale. This uncertainty budget can be applied to other kinds of reference thermometers, such as Sea Bird Electronics SBE 35, which are used to calibrate CTD profilers at sea. In the best case, it leads to a combined standard uncertainty of $0.39 \mathrm{mK}$. This value is largely dependent on the uncertainty of the temperature assigned to the reference cells, which is given by the primary calibration laboratories. For example, a gallium melting point cell calibrated in the UK with a UKAS certificate will have an expanded uncertainty of $\pm 0.25 \mathrm{mK}$. The same calibration made in France by the National Calibration Laboratory (LNE) under the same procedure, with a COFRAC certificate, will be given with an expanded uncertainty of $1.2 \mathrm{mK}$. This will give a combined standard uncertainty of $0.7 \mathrm{mK}$.

The drift of a reference thermometer between two annual calibrations can be equivalent to a standard uncertainty of $0.1 \mathrm{mK}$ and self-heating corrections can lead to a standard uncertainty of $0.2 \mathrm{mK}$. The stability and the uniformity of the temperature of the calibration bath can be evaluated by the shifts and standard deviations of data series measured at different places in the bath. It will express the reproducibility of measurements at all places in the bath. This reproducibility can be estimated, in the best case, for a seawater bath, as $0.3 \mathrm{mK}$ in the range $0-40^{\circ} \mathrm{C}$. 


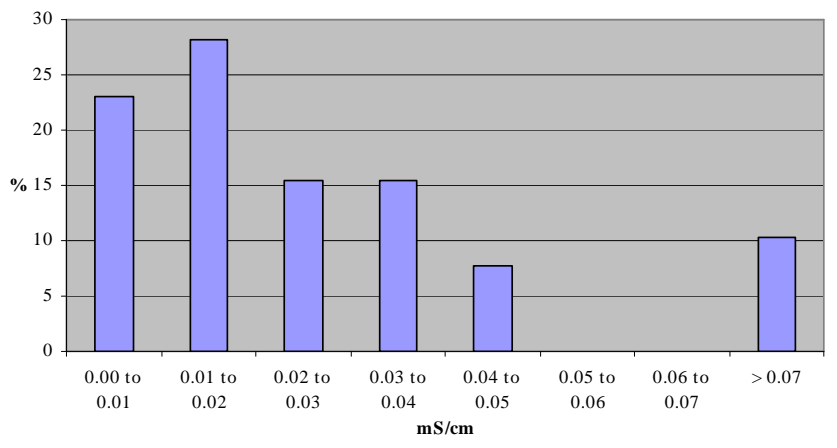

Fig. 2. Annual drift statistics of the 32 thermosalinographs SBE 21 of the French operational oceanography project Coriolis. Only $23 \%$ of them show annual shifts less than $0.01 \mathrm{mS} \mathrm{cm}^{-1}$ but $51 \%$ have annual shifts less than $0.02 \mathrm{mS} \mathrm{cm}^{-1}$. This shows how much drift there can be in conductivity cells submitted to strong environmental conditions.

Then, the reference temperature's combined uncertainty can be, in the best case: $u_{t}=0.54 \mathrm{mK}$. With a COFRAC certificate on the fixed points cell: $u_{t}=0.80 \mathrm{mK}$.

Table 3 shows values of $u_{\text {Cref }}$ calculated for different temperatures, conductivities and $S=35$, with $u_{t}=0.54 \mathrm{mK}$ and $u(S)=0.0011$. Monte Carlo assessments and GUM calculations give very close values, and it appears that, even with a very good measure of temperature, standard combined uncertainties of reference conductivities are close or superior to $0.002 \mathrm{mS} \mathrm{cm}^{-1}$ for high conductivity values. It also appears that the cross-term of the expression (17) cannot be neglected.

Conductivity sensors of CTD floats or profilers are linearized with polynomials before being used at sea. So, to find out what the uncertainty of the measured conductivity is, we must add to $u_{\text {Cref }}$, the square sum of the polynomial residuals $u_{C l}$ and the uncertainty on the CTD sensor readings which can be assessed by the repeatability of the sensor measurements $u_{C r} . u_{\mathrm{Cref}}, u_{\mathrm{Cl}}$ and $u_{\mathrm{Cr}}$ being independent variables, the expanded uncertainty $U_{C}$ of the conductivity values measured at sea (with a level of confidence close to $95 \%)$, can be expressed as:

$U_{C}=2 \sqrt{u_{\text {Cref }}^{2}+u_{C l}^{2}+u_{C r}^{2}}$

An estimate of the annual drift of the sensor could be added to this sum to have a real idea of the uncertainty of conductivity measurements. This error source has very variable amplitudes because it depends on the environment and the duration of measurements but also on the use of anti-fouling devices or on the regularity of sensor cleaning. It varies from $0.001 \mathrm{mS} \mathrm{cm}^{-1} \mathrm{yr}^{-1}$ to several $0.01 \mathrm{mS} \mathrm{cm}^{-1} \mathrm{yr}^{-1}$. In order to illustrate this variation, Fig. 2 shows the statistics of 32 thermosalinographs SBE 21 calibrated yearly at the SHOM calibration laboratory since 2003, in the framework of the French operational oceanography project Cori- olis, which contributes to the ARGO and GODAE experiences. This figure shows the drifts of conductivity cells submitted to strong environmental conditions.

So, in order to assess the value of $U_{C}$, we will just consider the initial uncertainty of the measurements. A usual value for $u_{C l}$ is $0.0002 \mathrm{mS} \mathrm{cm}^{-1}$ and, as an example, we can take the repeatability of a Sea Bird Electronics SBE 4 conductivity cell, which can be considered as equivalent to their resolution or $u_{C r}=0.0004 \mathrm{mS} \mathrm{cm}^{-1}$, and it follows a Normal law. $U_{C}$ has been calculated with these values and the results are also given in Table 3. It appears that expanded uncertainties of conductivity measured values, obtained with the two methods, are largely greater than $0.002 \mathrm{mS} \mathrm{cm}^{-1}$, particularly for high conductivity values.

\section{Uncertainties on salinities calculated from CTD sensors data}

Salinity is calculated with relation (9) when data are measured with CTD sensors, but the pressure effect must be taken into account and in this case, $R_{t}$ is obtained with:

$R_{t}=\frac{R}{r_{t} R_{p}}$

In this relation, $r_{t}$ is given by the relation (3) and its uncertainty by the relation (8), in which $u_{t}$ is the standard uncertainty of the temperatures measured by the CTD sensor. Considering the elements given in the previous paragraph about temperature calibration and the quality of the CTD's temperature sensors, $u_{t}$ can be estimated to be equal to $0.001^{\circ} \mathrm{C}$ (in the best case).

$R$ is the ratio:

$R=\frac{C(S, t, p)}{C(35,15,0))}$

$C(S, t, p)$ is the conductivity measured by the conductivity sensor. Its expanded uncertainty is given by the relation (18). $C(35,15,0)$ is the constant whose value has been discussed in the previous paragraph. $C(35,15,0)=42.914 \mathrm{mS} \mathrm{cm}^{-1}$ and $u_{R}=u_{C}=U_{C} / 2$, values of $U_{C}$ being given in Table 3 .

$R_{p}$ is the coefficient for pressure effects correction. $R_{p}$ is given by:

$R_{p}=1+\frac{p\left(e_{1}+e_{2} p+e_{3} p^{2}\right)}{\left(1+d_{1} t+d_{2} t^{2}+\left(d_{3}+d_{4} t\right) R\right)}$

$e_{1}, e_{2}, e_{3}$ and $d_{1}, d_{2}, d_{3}, d_{4}$ are constants whose values are given in Perkin and Lewis (1980). $p$ and $t$ are two independent quantities, but $R$ is proportional to $C(S, t, p)$ and strongly correlated to $t$. The calculation of the correlation coefficient $r_{R, t}$, with the temperature-conductivity data of Table 3 gives $r_{R, t}=0,9995$. Let us take $r_{R, t} \approx 1$. In this case, the combined standard uncertainty on $R_{p}\left(u_{R p}\right)$ can be written:

$u_{R_{p}}^{2}=\left(\frac{\partial R_{p}}{\partial p}\right)^{2} u_{p}^{2}+\left(\frac{\partial R_{p}}{\partial t} u_{t}+\frac{\partial R_{p}}{\partial R} u_{R}\right)^{2}$ 
Table 3. Standard combined uncertainties on reference conductivities $\left(u_{C \text { ref }}\right)$, computed for different values of temperature, conductivity and for $S=35$, and expanded uncertainty of conductivity values measured with linearized sensors $\left(U_{C}\right)$. $U_{C}$ and $u_{C}$ ref were assessed with the GUM and Monte Carlo Method (M.C.).

\begin{tabular}{cccccc}
\hline $\begin{array}{c}\text { Temperature } \\
\left({ }^{\circ} \mathrm{C}\right)\end{array}$ & $\begin{array}{c}\text { Conductivity } \\
\left(\mathrm{mS} \mathrm{cm}^{-1}\right)\end{array}$ & $\begin{array}{c}u_{C \mathrm{ref}}\left(\mathrm{mS} \mathrm{cm}^{-1}\right) \\
\text { GUM }\end{array}$ & $\begin{array}{c}u_{C \mathrm{ref}}\left(\mathrm{mS} \mathrm{cm}^{-1}\right) \\
\text { M.C. }\end{array}$ & $\begin{array}{c}U_{C}\left(\mathrm{mS} \mathrm{cm}^{-1}\right) \\
\text { GUM }\end{array}$ & $\begin{array}{c}U_{C}\left(\mathrm{mS} \mathrm{cm}^{-1}\right) \\
\text { M.C. }\end{array}$ \\
\hline 0 & 29.0360 & 0.0011 & 0.0012 & 0.0024 & 0.0025 \\
5 & 33.4554 & 0.0013 & 0.0013 & 0.0027 & 0.0028 \\
10 & 38.0897 & 0.0014 & 0.0015 & 0.0030 & 0.0030 \\
15 & 42.9175 & 0.0016 & 0.0016 & 0.0032 & 0.0032 \\
20 & 47.9180 & 0.0017 & 0.0017 & 0.0035 & 0.0036 \\
25 & 53.0710 & 0.0019 & 0.0019 & 0.0038 & 0.0039 \\
30 & 58.3570 & 0.0020 & 0.0020 & 0.0041 & 0.0042 \\
35 & 63.7569 & 0.0022 & 0.0021 & 0.0044 & 0.0044 \\
40 & 69.2527 & 0.0023 & 0.0024 & 0.0047 & 0.0048 \\
\hline
\end{tabular}

The calculation of the sensitivity coefficients leads us to write the final relation:

$$
\begin{aligned}
& u_{R_{p}}= \\
& \frac{\left[\left(e_{1}+2 e_{2} p+3 e_{3} p^{2}\right)^{2} u_{p}^{2}+\left(R_{p}-1\right)^{2}\left[\left(d_{1}+2 d_{2} t+d_{4} R\right) u_{t}+\left(d_{3}+d_{4} t\right) u_{R}\right]^{2}\right]^{1 / 2}}{\left(1+d_{1} t+d_{2} t^{2}+\left(d_{3}+d_{4} t\right) R\right)}
\end{aligned}
$$

The value of $u_{p}$, the standard uncertainty of pressure measurements, remains to be found. Accuracy and precision of pressure sensors depend on their range of measurement. Pressure balances used to calibrate them, must undergo corrections for normal gravity, height difference with the sensor, thermal and pressure expansion of the piston and of the air-mass hydrostatic pressure difference. After that, the expanded uncertainty of a reference pressure given by an 8000 dbar balance is calculated by a relation of this kind:

$U_{P \text { ref }}=0.12+0.00013 p(\mathrm{dbar})$

If the repeatability of a $6000 \mathrm{dbar}$ sensor (and its electronics) is $0.2 \mathrm{dbar}$, and that its residual temperature drift is also $0.2 \mathrm{dbar}$, then $u_{p}=0.53 \mathrm{dbar}$ at $6000 \mathrm{dbar}$ or $0.34 \mathrm{dbar}$ at 2000 dbar.

With these elements, the expression of the standard combined uncertainty on $R_{t}$, obtained with relation (19) must still be written. So, the GUM method applied to relation (19) leads us to write:

$$
\begin{aligned}
u_{\mathrm{c}}\left(R_{t}\right)^{2}= & \left(\frac{\partial R_{t}}{\partial R}\right)^{2} u_{R}^{2}+\left(\frac{\partial R_{t}}{\partial R_{p}}\right)^{2} u_{R_{p}}^{2}+\left(\frac{\partial R_{t}}{\partial r_{t}}\right)^{2} u_{r_{t}}^{2} \\
& +2 \frac{\partial R_{t}}{\partial R} \frac{\partial R_{t}}{\partial R_{p}} u_{R} u_{R_{p}} r_{R, R_{p}}+2 \frac{\partial R_{t}}{\partial r_{t}} \frac{\partial R_{t}}{\partial R_{p}} u_{r_{t}} u_{R_{p}} r_{r_{t}, R_{p}} \\
& +2 \frac{\partial R_{t}}{\partial R} \frac{\partial R_{t}}{\partial r_{t}} u_{R} u_{r_{t}} r_{R, r_{t}}
\end{aligned}
$$

The development of the relation (25) gives:

$u_{\mathrm{c}}\left(R_{t}\right)=R_{t}\left[\left(\frac{u_{R}}{R}\right)^{2}+\left(\frac{u_{R_{p}}}{R_{p}}\right)^{2}+\left(\frac{u_{r_{t}}}{r_{t}}\right)^{2}-2 \frac{u_{R}}{R} \frac{u_{R_{p}}}{R_{p}} r_{R, R_{p}}\right.$

$$
\left.+2 \frac{u_{R_{p}}}{R_{p}} \frac{u_{r_{t}}}{r_{t}} r_{r_{t}, R_{p}}-2 \frac{u_{R}}{R} \frac{u_{r_{t}}}{r_{t}} r_{R, r_{t}}\right]^{1 / 2}
$$

The correlation coefficients of the variables $R, R_{t}$ and $r_{t}$ have been computed for the salinities $S=10,35,38$ and 40 , with the numerical values of $t, C$ and $p$ displayed in Table 4. This gives: $r_{R, R p}=-0.44, r_{R, r t}=0.998 \approx 1$ and $r_{R p, r t}=-0.50$, and shows that cross-terms cannot be neglected. Moreover, neglecting this terms would increase the uncertainty estimate. For example, for $t=2{ }^{\circ} \mathrm{C}, C=$ $33.038 \mathrm{mS} \mathrm{cm}^{-1}$ and $p=6000 \mathrm{dbar}, u_{\mathrm{c}}\left(R_{t}\right)=5 \times 10^{-4}$ and $u_{\mathrm{c}}(S)=0.0019$, without the correlation terms, but with this terms $u_{\mathrm{c}}\left(R_{t}\right)=1.4 \times 10^{-5}$ and $u_{\mathrm{c}}(S)=0.0005$ ! The uncertainties on practical salinity computations take advantage of the ratio expression of $R_{t}$, which reduces the effect of the uncertainties of each of the input variables.

With the correlation coefficients given previously, relation (26) can be simplified to give:

$u_{\mathrm{c}}\left(R_{t}\right)=R_{t}\left[\left(\frac{u_{R}}{R}-\frac{u_{r_{t}}}{r_{t}}\right)^{2}+\frac{u_{R_{p}}}{R_{p}}\left(\frac{u_{R_{p}}}{R_{p}}+0.88 \frac{u_{R}}{R}-\frac{u_{r_{t}}}{r_{t}}\right)\right]^{1 / 2}$

In fact, it is the standard uncertainty of the PSS-78 relations fits, given in Perkin and Lewis (1980), which increases the uncertainty in $S$ significantly, particularly in the case when $R_{p}$ value is different from 1: $u_{\text {PSS }}$ is then equal to 0.0015 .

Then, in the case of CTD measurements, the expanded uncertainty in salinity computations can be assessed (with a level of confidence close to $95 \%$ ) by the relation:

$U_{S}=2 \sqrt{u_{\mathrm{c}}(S)^{2}+u_{\mathrm{PSS}}^{2}}$

where $u_{\mathrm{c}}(S)$ can be calculated with relations (13) and (26) or (27).

Table 4 shows the expanded uncertainties of practical salinities calculated from relation (28) using the Monte Carlo method. The two methods give equivalent results 
Table 4. Expanded combined uncertainties on salinity, computed with representative values of temperature, conductivity and pressure and their combined standard uncertainties. Conductivity combined standard uncertainties $u_{C}$ correspond to the values found in table 3 and for temperature, the standard uncertainty corresponds to the best case when $u_{t}=0.001{ }^{\circ} \mathrm{C}$. Idem for $u_{p}$.

\begin{tabular}{ccccccccc}
\hline $\begin{array}{c}\text { Temperature } \\
\left({ }^{\circ} \mathrm{C}\right)\end{array}$ & $\begin{array}{c}\text { Conductivity } \\
\left(\mathrm{mS} \mathrm{cm}^{-1}\right)\end{array}$ & $\begin{array}{c}\text { Pressure } \\
(\mathrm{dbar})\end{array}$ & $\begin{array}{c}u_{t} \\
\left({ }^{\circ} \mathrm{C}\right)\end{array}$ & $\begin{array}{c}u_{C} \\
\left(\mathrm{mS} \mathrm{cm}^{-1}\right)\end{array}$ & $\begin{array}{c}u_{p} \\
(\mathrm{dbar})\end{array}$ & $\begin{array}{c}U_{S} \\
(\mathrm{GUM})\end{array}$ & $\begin{array}{c}U_{S} \\
(\mathrm{M} . \mathrm{C} .)\end{array}$ \\
\hline 15 & 13.7031 & 0 & 0.001 & 0.0012 & 0.29 & 10.000 & 0.0033 & 0.0033 \\
0 & 29.0360 & 0 & 0.001 & 0.0012 & 0.29 & 35.000 & 0.0032 & 0.0032 \\
35 & 71.7249 & 0 & 0.001 & 0.0025 & 0.29 & 40.000 & 0.0034 & 0.0034 \\
40 & 69.2527 & 0 & 0.001 & 0.0024 & 0.29 & 35.000 & 0.0034 & 0.0034 \\
15 & 42.9175 & 0 & 0.001 & 0.0016 & 0.29 & 35.000 & 0.0032 & 0.0033 \\
12 & 40.2209 & 500 & 0.001 & 0.0016 & 0.30 & 35.000 & 0.0033 & 0.0033 \\
10 & 38.5295 & 1000 & 0.001 & 0.0015 & 0.31 & 35.000 & 0.0032 & 0.0033 \\
5 & 34.3185 & 2000 & 0.001 & 0.0014 & 0.34 & 35.000 & 0.0032 & 0.0032 \\
4 & 34.1673 & 4000 & 0.001 & 0.0014 & 0.43 & 35.000 & 0.0032 & 0.0033 \\
3 & 33.6111 & 5000 & 0.001 & 0.0013 & 0.48 & 35.000 & 0.0032 & 0.0032 \\
2 & 33.0378 & 6000 & 0.001 & 0.0013 & 0.53 & 35.000 & 0.0032 & 0.0032 \\
\hline
\end{tabular}

( $U_{S}=0.0034$ on average). These results are greater by about 0.0014 than the 0.002 expected by the WOCE programme.

More, this uncertainty assessment is valid only in areas where temperature and salinity gradients are low. When measurements are made in areas of strong temperature and (or) salinity gradients, the major errors in practical salinity measurements come from the ability to align the response times of temperature and conductivity sensors, even when data are corrected with manufacturers' correction algorithms, as shown by Mensah et al. (2009). On average, errors up to 0.017 still persist for some measurements in strong salinity gradients and increase the uncertainty in practical salinity by as much, if they cannot be detected and corrected.

Lastly, we must not forget that practical salinity is only one way to approach the absolute salinity $S_{\mathrm{A}}$ of seawater which is the real quantity to access thermodynamic properties of the ocean and ocean-atmosphere interactions. Therefore, the fact that non-electrolyte components are not detected by conductivity sensors and that the seawater components ratio is not clearly known, leads to a difference of about $0.45 \pm 0.05 \%$ between $S$ and $S_{\mathrm{A}}$ and to an uncertainty of $0.16 \mathrm{ppt}$ in $S_{\mathrm{A}}$, even at $S=35$, as estimated by Jackett and McDougall (2006). Then, the expanded uncertainty of 0.0034 on $S$, as obtained with relation (28), can be considered as largely sufficient and even insignificant in the assessment of the absolute salinity.

\section{Conclusion}

The uncertainties of practical salinity calculations have been assessed by two standardized independent methods: the GUM and Monte Carlo, in the case of salinities obtained with laboratory salinometers and in the case of CTD measurements after laboratory calibration of conductivity cells.
The two methods give coherent and very similar results. The 0.002 psu required initially by the WOCE program are obtained with difficulty, even in the case of laboratory salinometers. However, in the error budget, the part due to the PSS-78 relations fits is sometimes as significant as the instrument's. This is particularly the case with CTD measurements where correlations between the $R_{t}$ variables contribute mainly to decreasing the uncertainty on $S$, even when the expanded uncertainties of conductivity cell calibrations are for the most part in the order of $0.002 \mathrm{mS} \mathrm{cm}^{-1}$. The relations given in this publication and obtained with the normalized GUM method, allow a real analysis of uncertainty sources and they can be used in a general way to assess the uncertainty in conductivity cells calibrations or practical salinity calculations made with data from instruments having specifications different from the examples taken in Tables 1 to 4 .

\section{Appendix A}

\section{PSS 78 algorithm as defined in Fofonoff and Millard (1983)}

$p$ is expressed in dbar, $t$ in ${ }^{\circ} \mathrm{C}$ and $C$ in $\mathrm{S} \mathrm{m}^{-1}$

$$
\begin{aligned}
& a_{1}=0.0080, a_{2}=-0.1692, a_{3}=25.3851, a_{4}=14.0941, \\
& a_{5}=-7.0261, a_{6}=2.7081
\end{aligned}
$$

$$
\begin{aligned}
& b_{1}=0.0005, \quad b_{2}=-0.0056, \quad b_{3}=-0.0066, \\
& b_{4}=-0.0375, b_{5}=0.0636, b_{6}=-0.0144 \\
& c_{1}=0.676697, c_{2}=2.00564 \times 10^{-2}, c_{3}=1.104259 \times \\
& 10^{-4}, c_{4}=-6.9698 \times 10^{-7}, c_{5}=1.0031 \times 10^{-9}
\end{aligned}
$$




$$
\begin{aligned}
& d_{1}=3.426 \times 10^{-2}, \quad d_{2}=4.464 \times 10^{-4}, d_{3}=0.4215, \\
& d_{4}=-3.107 \times 10^{-3} \\
& e_{1}=2.070 \times 10^{-5}, \quad e_{2}=-6.370 \times 10^{-10}, \quad e_{3}= \\
& 3.989 \times 10^{-15} \\
& R=C / 4.2914 \\
& R_{1}=c_{1}+\left(c_{2}+\left(c_{3}+\left(c_{4}+c_{5} \times t\right) \times t\right) \times t\right) \times t \\
& R_{P}=1+\left(\left(e_{1}+\left(e_{2}+e_{3} \times p\right) \times p\right) \times p\right) /\left(1+\left(d_{1}+d_{2} \times\right.\right. \\
& \text { t) } \left.\times t+\left(d_{3}+d_{4} \times t\right) \times R\right) \\
& R_{t}=R /\left(R_{1} \times R_{P}\right)
\end{aligned}
$$

Edited by: J. M. Huthnance

\section{References}

Bacon, S., Culkin, F., Higgs, N., and Ridout, P.: IAPSO Standard Seawater: definition of the uncertainty in the calibration procedure and stability of recent batches, J. Atmos. Oceanic Technol., 24, 1785-1799, 2007.

BIPM: Evaluation of measurement data - Supplement 1 to the Guide to the expression of uncertainty in measurement - Propagation of distributions using a Monte Carlo method, JCGM YYY:2006, 2006.

BIPM: Evaluation of measurement data - Guide to the expression of uncertainty in measurement, JCGM 100:2008, GUM 1995 with minor corrections, 2008.

Culkin, F. and Ridout, P. S.: Stability of IAPSO Standard Seawater, J. Atmos. Oceanic Technol., 15, 1072-1075, 1998.

Culkin, F. and Smith, N.: Determination of the concentration of potassium chloride solution having the same electrical conductivity, at $15^{\circ} \mathrm{C}$ and infinite frequency, as standard seawater of salinity 35,000\%o, (Chlorinity $19.37394 \%$ ), IEEE J. Oceanic Eng., OE-5, no. 1, 22-23, 1980.
Fellmuth, B., Fisher, J., and Tegeler, E.: Uncertainty budgets for characteristics of SPRTs calibrated according to the ITS-90, BIPM, CCT/01-02, 2002.

Fofonoff, N. P. and Millard, R. C.: Algorithms for computation of fundamental properties of seawater, Unesco technical paper in marine science 44, 1983.

Jackett, D. R. and McDougall, T. J.: Algorithms for density, potential temperature, conservative temperature and the freezing temperature of seawater, J. Atmos. Oceanic Technol., 23, 17091728, 2006.

Kawano, T., Aoyama, M., and Takatsuki, M.: Inconsistency in the conductivity of standard potassium chloride solutions made from different high-quality reagents, Deep Sea Res. Part. I, 52, 389396, 2005.

Lueck, R. G.: Thermal inertia of conductivity cells: theory, J. Atmos. Oceanic Technol., 7, 741-755, 1990.

Mensah, V., Le Menn, M., and Morel, Y.: Thermal mass correction for the evaluation of salinity, J. Atmos. Oceanic Technol., 26, 665-672, 2009.

Perkin, R. G. and Lewis, E. L.: The Practical Salinity Scale 1978: Fitting the Data, IEEE J. Oceanic Eng., OE-5, no. 1, 9-16, 1980.

Poisson, A.: Conductivity/Salinity/Temperature relationship of diluted and concentrated standard seawater, IEEE J. Oceanic Eng., OE-5, no. 1, 41-50, 1980.

Saunders, P. M., Mahrt, K.-H., and Williams, R. T.: Standard and Laboratory Calibration, WHP Operations and Methods, 1991.

Seitz, S., Spitzer, P., and Brown, R. J. C.: Consistency of practical salinity measurements traceable to primary conductivity standards: Euromet project 918, Accreditation and Quality Assurance, 13, 601-605, 2008.

Seitz, S., Spitzer, P., and Brown, R. J. C.: CCGM-P111 study on traceable determination of practical salinity and mass fraction of major seawater components, Accred. Qual. Assur., 15, 9-17, 2010.

Uschida, H., Kawano, T., and Fukasawa, M.: In situ calibration of moored CTDs used for monitoring abyssal water, J. Atmos. Oceanic Technol., 25, 1695-1702, 2008. 